\title{
Squamous Cell Carcinoma of the External Auditory Canal: Case Report
}

\author{
Zouhair N*, Chaouki A, Chafiki Z, Hasnaoui J, Abada R, Rouadi S, Roubal M and Mahtar M \\ Department of Otorhinolaryngology, Hospital of 20 august, Casablanca, Morocco
}

${ }^{*}$ Corresponding author: Zouhair N, Department of Otorhinolaryngology, Hospital of 20 august, Casablanca, Morocco, E-mail: najib_zouhair@hotmail.fr

Citation: Zouhair N, Chaouki A, Chafiki Z, Hasnaoui J, Abada R, et al. (2019) Squamous Cell Carcinoma of the External Auditory Canal: Case Report. J Case Rep Stud 7(1): 106. doi: 10.15744/2348-9820.7.106

Received Date: November 04, 2018 Accepted Date: February 26, 2019 Published Date: February 28, 2019

\begin{abstract}
Squamous cell carcinoma of the external auditory canal may masquerade as an infective process causing late diagnosis. A delay in treatment as a result of missed diagnosis would carry a poor prognosis as the disease progresses to an advanced stage. We present a case of a man with history of chronic otorrhea, who developed left sided otalgia associated with hearing loss. otoscopic examination shows a mass in her left ear and pus discharge which was initially treated as an infection. The biopsy of the mass was proven to be squamous cell carcinoma. High index of suspicion is necessary when encountering patients presenting with a mass in the ear canal with prior history of chronic otorrhea or cholesteatoma. Proper tissue biopsy is crucial. Early diagnosis is required for better prognosis for the patient.
\end{abstract}

Keywords: Squamous Cell Carcinoma; External Auditory Canal; Misdiagnosis; Biopsy; Low Prognostic

\section{Introduction}

Temporal bone carcinoma is rare, occurring about $0.2 \%$ of all cancer of the head and neck [1]. Histologically it can arise from epithelial, mesenchymal or salivary gland. Eighty-six percent of these tumors are squamous cell carcinoma (SCC) [2]. The most common symptoms of this malignancy are ear pain and discharge [3]. These symptoms are similar to those of chronic suppurative otitis media and otitis externa and therefore SCC is easily misdiagnosed. Late diagnosis of SCC of the temporal bone is common, which worsens the prognosis [4].

\section{Materials and Methods}

Case study about a patient which was treated in ENT unit of 20 Augusts' hospital, Casablanca, Morocco until November 2016. Information's was collected using patients folder and operations register, photos taken from the informatics unit of the department.

\section{Results}

A 41-year-old man presented with 1-year history of discharge from the left ear. His symptom was associated with mild pain in the left ear, tinnitus and loss of hearing. He was treated with an antibiotic ear drop at a private clinic. On his subsequent visit to the private clinic with similar symptoms, he was given an antibiotic ear drop and referred to an otorhinolaryngologist for the follow-up treatment. He had no rhinitis symptoms. On otoscopic examination, an exophytic mass occupying external auditory canal of left ear with minimal pus was observed (Figure 1). His tympanic membrane was not visualised and no facial nerve palsy. Audiometric test showed left conductive hearing loss: at $75 \mathrm{db}$ CT scan showed filling tissular of the meatus, attic and tympanic membrane of the left ear, so then bone lysis of ear ossicles, tegmen tympani, antrum and extension to the skull base, in addition he has lysis of the outer wall of the lateral semicircular canal and facial canal in its tympanic portion (Figure 2).

At surgical exploration we found a very extensive process attained meanings, mastoid and posterior cerebral fosse biopsies were done, and patient was referred to oncologist for radiotherapy.

Biopsy of the mass from His left ear was done. The histopathology examination of the biopsy showed invasive well-differentiated SCC with keratin pearls. The tumor cells were arranged in sheets and trabecular pattern. The diagnosis was SCC of the external auditory canal. 


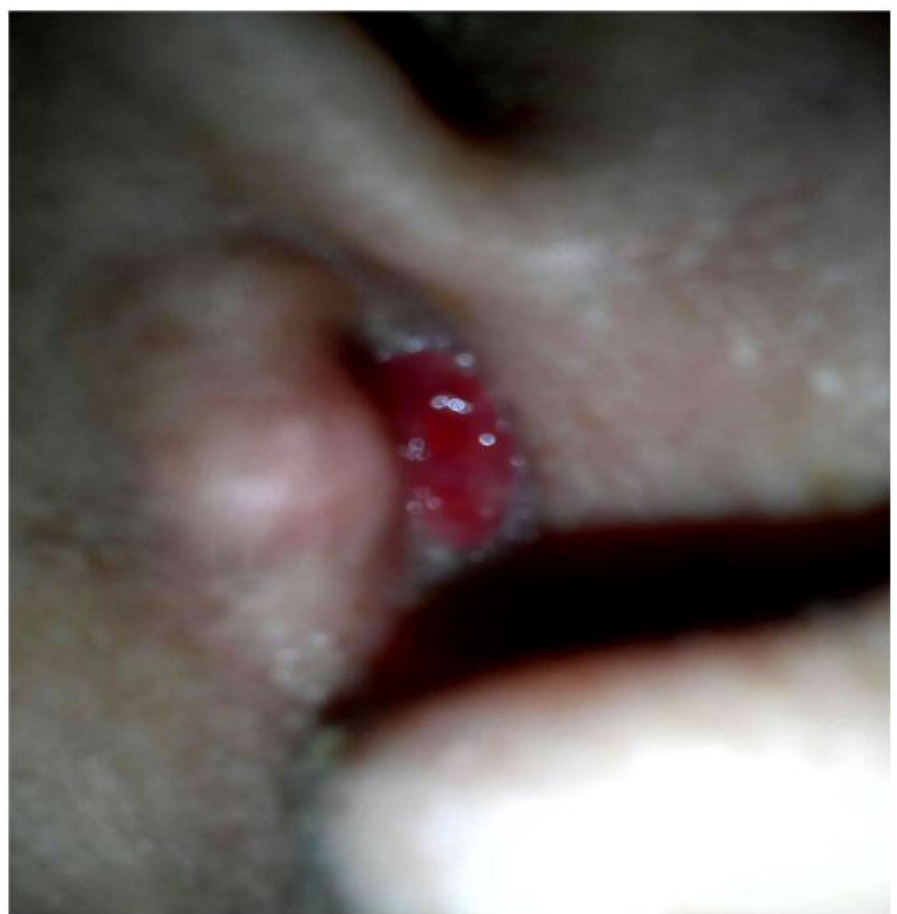

Figure 1: Exophytic mass occupying external auditory canal of left ear (the arrow)

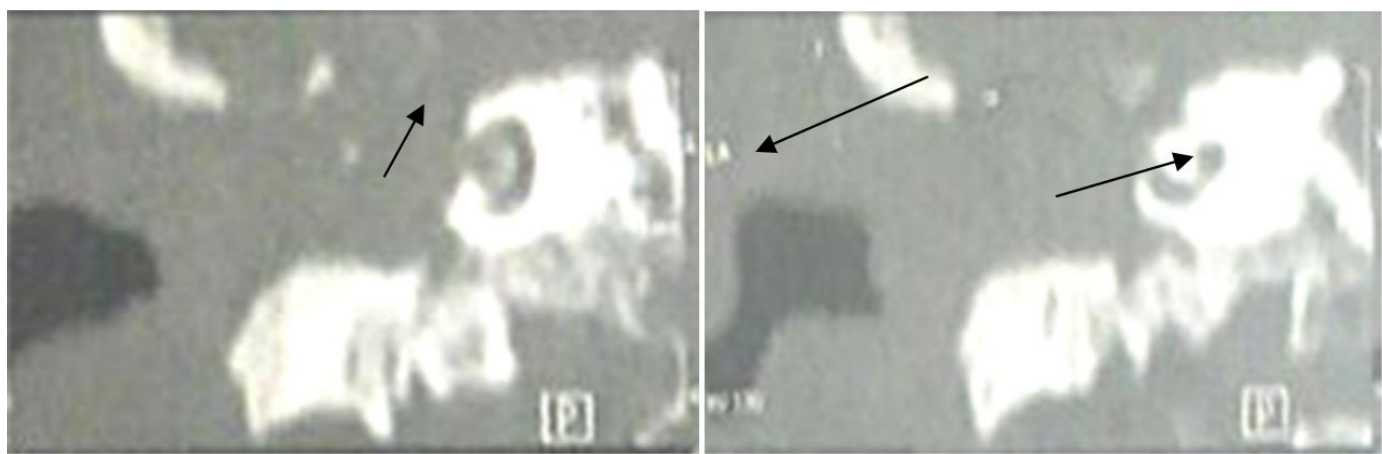

Figure 2: CT scan showed extensive process with tegmen lysis semicircular canal outer wall lysis (arrows)

\section{Discussion}

There might be an unusual localization of SCC at times. The carotid canal, jugular foramen, dura, middle and posterior cranial fossae are involved in advanced stages [5]. SCC of the temporal bone has a slightly higher prevalence in women than men [6]. The median age at presentation of SCC of the temporal bone is in the seventh decade [7]. The clinical features of SCC of the temporal bone are non-specific.

Therefore, most of the cases were misdiagnosed, especially in the early stages. The most common misdiagnosis is otitis external; others include chronic supportive otitis media, granulation in external auditory canal, cholesteatoma and papilloma. In our aforementioned context, the patient was thought to have chronic suppurative otitis media initially and was repeatedly prescribed an antibiotic ear drop by the medical doctor in the private clinic.

The presence of ear bleeding can be due to granulation tissue in the external auditory canal. This tumour is often associated with chronic otitis media and exposure to radiation therapy [8]. In case of non-resolving ear discharge, especially with the presence of external auditory mass, there must be a suspicion towards the possibility of malignancy. Some suggest to do biopsy in patients with longstanding ear infection, who are not responsive to standard therapy. Biopsy must not be superficial, as this may lead to misdiagnosis [1,2]. The reliability of biopsy depends on the location and depth of the specimen collected [9]. Due to the rarity of the SCC of the temporal bone, developing an adequate staging system and treatment is difficult. To date, modified Pittsburgh staging system is used for staging purpose [10]. The preferred treatment for this tumour is the en bloc surgical resection of the primary tumour with tumour-free surgical margins, postoperative chemotherapy and radiotherapy. The 2-year survival rates reported for patients who have undergone surgical resection with or without adjuvant radiotherapy vary considerably by study [11]. Poor prognostic factors include extent of disease at presentation, dural and cranial nerve involvement, facial nerve paralysis and positive margin [8]. 


\section{Conclusion}

Every physician therefore must suspect temporal bone malignancy, especially in longstanding ear infection that does not respond to standard therapy. Biopsy must be performed and additionally, imaging should be done to see the extension of the tumour. Dedication, professionalism and careful examination of divergent and specific patients are thoroughly advised.

\section{References}

1. Antonio SM (2007) Malignant Tumours of the Temporal Bone. eMedicine Otolaryngology.

2. Arriaga MA, Hall C, Nuss D, Kunduk M (2007) Salivary Gland Disorders: Temporal Bone Resection. Springer Berlin Heidelberg $23: 393-405$.

3. Gidley PW, DeMonte F (2013) Temporal bone malignancies. Neurosurg Clin N Am 24: 97-110.

4. Al-Shidhabi A (1992) Carcinoma of temporal bone presenting as malignant otitis externa. J Laryngol Otol 908-10.

5. Leonetti JP, Smith PG, Kletzker GR (1996) Invasion patterns of advanced temporal bone malignancies. Am J Otol 17: 438-42.

6. Morton RP, Stell PM, Derrick PP (1984) Epidemiology of cancer of the middle ear cleft. Cancer 53: 1612-7.

7. Kinney SE, Wood BG (1987) Malignancies of the external ear canal and temporal bone: Surgical techniques and results. Laryngoscope 97: 158-64.

8. Lobo D, Llorente JL, Suarez C (2008) Squamous cell carcinoma of the external auditory canal. Skull Base 18: 167-72.

9. Zhen S, Fu T, Qi J (2014) Diagnosis and treatment of carcinoma in external auditory canal. J Otol 9: 146-50.

10. Moody SA, Hirsch BE, Myers EN (2000) Squamous cell carcinoma of the external auditory canal: An evaluation of a staging system. Am J Otol 21: 582-8.

11. Prasad SC, D'Orazio F, Medina M, Bacciu A, Sanna M (2014) State of the art in temporal bone malignancies. Curr Opin Otolaryngol Head Neck Surg 22: 154-65. 